\title{
The dune size distribution and scaling relations of barchan dune fields
}

\author{
Orencio Durán • Veit Schwämmle • Pedro G. Lind • \\ Hans J. Herrmann
}

Received: 19 May 2008 / Published online: 26 November 2008

(c) The Author(s) 2008. This article is published with open access at Springerlink.com

\begin{abstract}
Barchan dunes emerge as a collective phenomena involving the generation of thousands of them in so called barchan dune fields. By measuring the size and position of dunes in Moroccan barchan dune fields, we find that these dunes tend to distribute uniformly in space and follow an unique size distribution function. We introduce an analytical mean-field approach to show that this empirical size distribution emerges from the interplay of dune collisions and sand flux balance, the two simplest mechanisms for size selection. The analytical model also predicts a scaling relation between the fundamental macroscopic properties characterizing a dune field, namely the inter-dune spacing and the first and second moments of the dune size distribution.
\end{abstract}

\section{O. Durán $(\varangle)$}

Institute for Computational Physics, Stuttgart University, 70569 Stuttgart, Germany

e-mail: o.duran@utwente.nl; o.duran@ctw.utwente.nl

Present Address:

O. Durán

Multi Scale Mechanics (MSM), Twente University,

7500 AE Enschede, The Netherlands

\section{Schwämmle}

Centro Brasileiro de Pesquisas Físicas,

22290-180 Urca, Rio de Janeiro (RJ), Brazil

P. G. Lind

Centro de Fsica Terica e Computacional, Universidade de Lisboa

Avenida, Professor Gama Pinto 2, 1649-003 Lisbon, Portugal

\section{H. J. Herrmann}

Computational Physics,

IfB, HIF E12, ETH Hönggerberg, 8093 Zurich, Switzerland

\section{H. J. Herrmann}

Departamento de Física, Universidade Federal do Ceará, 60451-970 Fortaleza, Ceará, Brazil
Keywords Pattern formation - Dune fields - Dune collisions $\cdot$ Master equation $\cdot$ Log-normal distributions

\section{Introduction}

A first glance over an extensive desert area shows not only that dunes are ubiquitous and present well-selected shapes, but also that they typically emerge in groups with a very well defined characteristic dune size and inter-dune spacing, forming fields of up to several thousands of dunes (see Fig. 1a-d). These observations naturally rise questions concerning the way dunes distribute throughout the deserts. What are the mechanisms of the size selection process behind such uniformity? Do the size distributions of such dune fields follow a simple unique function or do they depend on the local conditions?

Sand dunes have been intensively studied in the last years. It is now well-understood what are the fundamental laws underlying the emergence of one single barchan dune and what mechanisms maintain its shape while moving [1-3]. For instance, barchans occur in areas with unidirectional wind and low sand availability. The influence of the geographical constrains and the external physical conditions [2-4], of the dune-dune interactions [5-7] and even of the emergence of vegetation covers [8] in the dynamics and morphology of single dunes were quite well-established with the help of dune models $[1,8,9]$. There are also a few studies of entire dune fields [10-13], but a simple theoretical understanding of the size selection process within dune fields has still not been achieved.

In this work, we present a first answer to this problem. First, we show that, while a single dune is suitably characterized by its width $w[2,3,13]$, an entire dune field contains dunes with different sizes following a unique distribution 

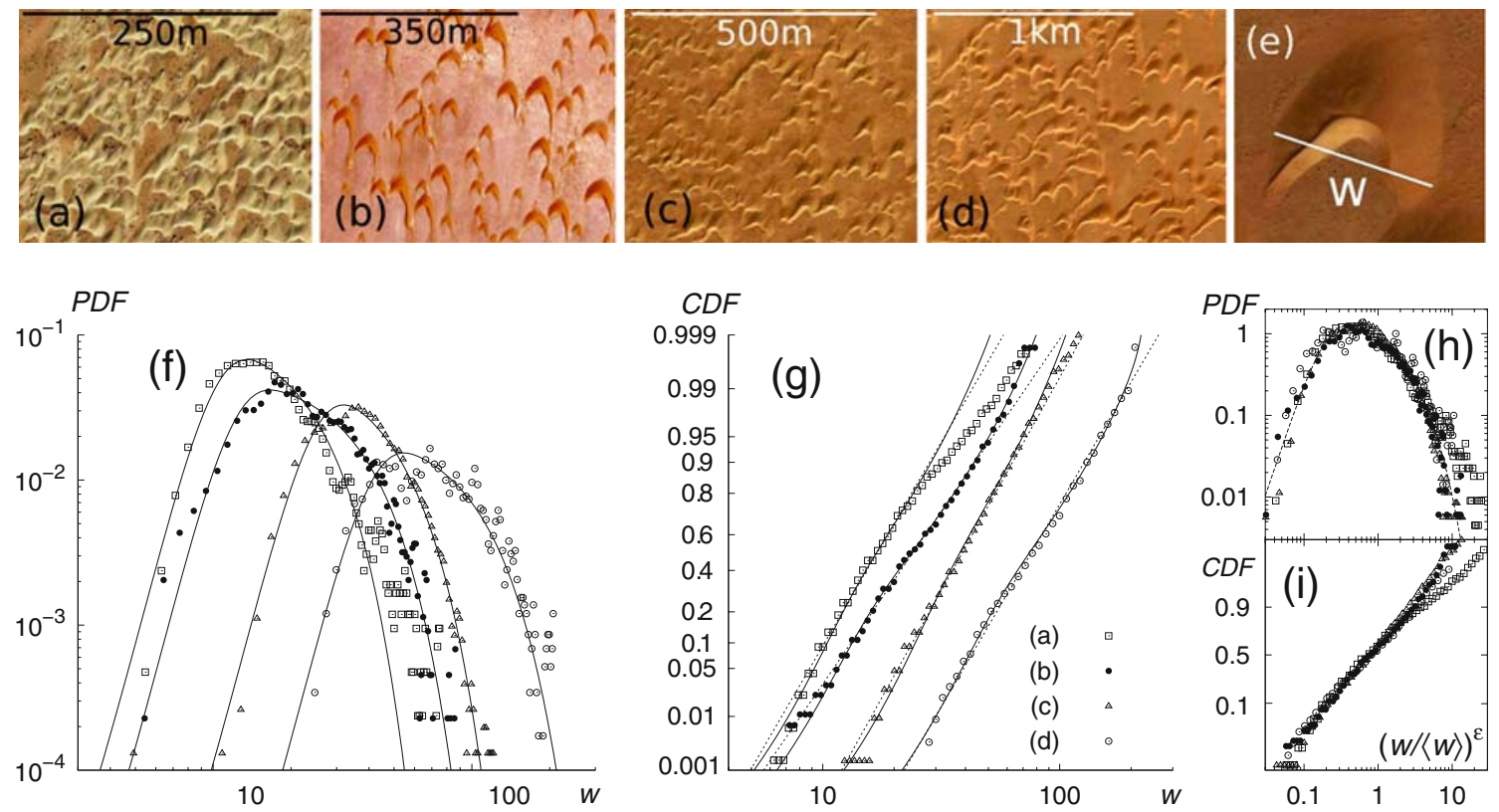

Fig. 1 Details of barchan dune fields in Morocco, Western Sahara. The number of measured dunes is 1,295 (a), 1,113 (b), 1,947 (c) and 1,630 (d), covering areas of $\sim 3,7,12$ and $60 \mathrm{~km}^{2}$ and with average dune sizes of 17,27, 42 and $86 \mathrm{~m}$, respectively. The size of a barchan dune is characterized by its width $w(\mathbf{e})$. In all pictures, the North points up. Images provided by GoogleEarth. f Probability density function $(P D F)$ of the dune size for the measured Moroccan dune fields (symbols) and the best fit using the analytical solution (solid lines) given by (1). $\mathrm{g}$ Cumulative

(Fig. 1f, g). Consequently, the corresponding average $\langle w\rangle$ and standard deviation $S=\sqrt{\left\langle w^{2}\right\rangle-\langle w\rangle^{2}}$ are suitable properties to characterize the field. Additionally to these two quantities, we show that the inter-dune spacing $L$ is also a property with characteristic values $\langle L\rangle$ and therefore also able to characterize the field. Second, using numerical simulations, we show that collisions between dunes play a crucial role in the selection of a characteristic dune size. Finally, from a mean-field approach that couples the effect of dune collision with that of sand flux balance, we derive the size distribution function (shown in Fig. 1f, g) and a scaling relation between the three properties of the field, $\langle L\rangle,\langle w\rangle$ and $S$.

\section{Measurements of the dune size and inter-dune spacing distribution}

We start by measuring the width (Fig. 1e) of more than 5,000 dunes composing four dune fields located in the Western Sahara (Fig. 1a-d). For all fields, the dune width distribution exhibits a unique function, apart small deviations at the extremes $(w \lesssim 10 \mathrm{~m}$ and $w \gtrsim 0.5 \mathrm{~km}$ [13]), as shown in Fig. 1f, g. This function will be derived later. The relative broadness $S /\langle w\rangle$ scales with the relative inter-dune spacing distribution function $(C D F)$ with the analytical solution (solid lines) and the log-normal straight-line for reference (dashed lines). The relative broadness $S /\langle w\rangle$ is given by the inverse of the slope of the CDF. After rescaling the dune sizes as $(w /\langle w\rangle)^{\epsilon}$ with $\epsilon=2.9 \sqrt[3]{\langle w\rangle /\langle L\rangle}$, all PDFs and CDFs in $\mathbf{h}$ and $\mathbf{i}$, respectively collapse, uncovering a scale invariance between the size distributions of different barchan dune fields. In $\mathbf{h}$ the analytical distribution (see text) is also shown (dashed line), as an eye-guide

as $\sqrt[3]{\langle L\rangle /\langle w\rangle}$, as shown in Fig. 1h, i. This scaling law relates the spatial distribution of dunes and their size distribution and will also be deduced later as a result of the size selection model we propose. Therefore, the mechanisms leading to such distributions should not depend on the absolute size and inter-dune spacing of the dunes involved. Instead, they should depend on the relative dune size and spacing. In other words, they should be scale invariant.

In barchan dune fields, the sand flux balance on single dunes leads to an instability in the dune size [7]. Dunes nucleate as a consequence of the sand accumulation along the field and no characteristic size emerges $[7,11]$. However, the peaked size distributions in Fig. If are found in dune fields where sand flux balance is not the only process mediating the size of dunes. Since barchan dunes move over the field with velocities that strongly depend on the size $(v \propto 1 / w)$ [1], collisions are ubiquitous in such fields, turning out to be another relevant process for dune size alterations $[7,13]$. Therefore, the dune size distribution should be determined by the competition between the balance of sand flux on a single dune and the collisions between neighboring dunes $[6,7,11]$. Recently a third size selection mechanism was discovered, that involves the calving of large dunes due to wind fluctuations $[13,14]$. This is a complex scale dependent process, relevant for fields with large dunes and should not be 

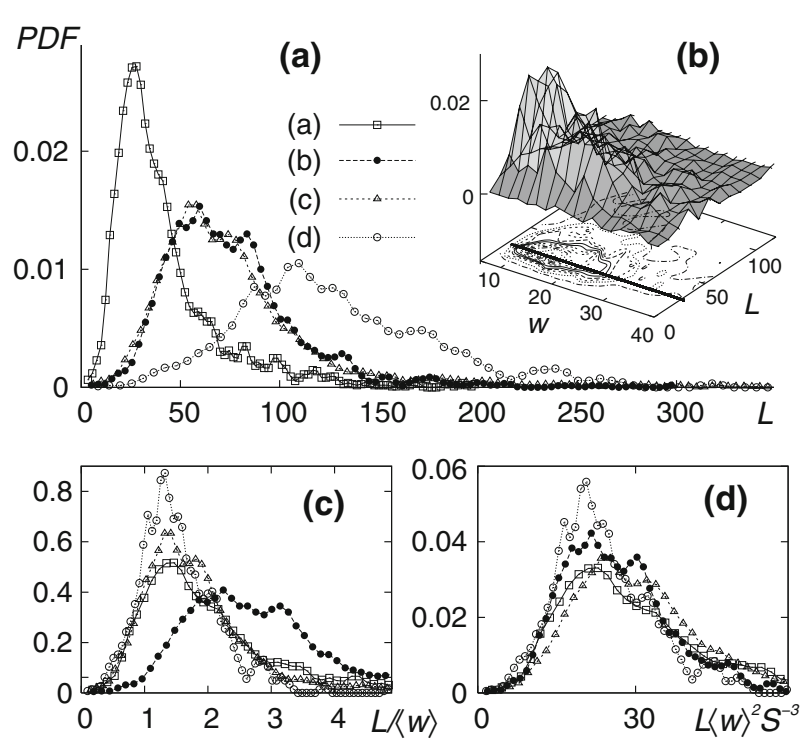

Fig. 2 a The PDF of the inter-dune spacing clearly shows a characteristic value $\langle L\rangle$ for each dune field depicted in Fig. 1a-d. The inter-dune spacing $L$ around a given dune is defined as the square root of the empty area of a polygon formed by the centers of the nearest dunes, one in each quadrant of the Cartesian frame reference centered at the dune. b PDF of $L$ as a function of the dune width $w$ for the first dune field (a). From the contour plot, one defines the characteristic inter-dune spacing $\langle L\rangle$ (solid line), taken as the average over the highest frequency region, which is independent of $w$ (see text). c After rescaling $L$ by the mean dune size $\langle w\rangle$, not all PDFs peak in the same relative inter-dune spacing $\langle L\rangle /\langle w\rangle$. d However, the curves collapse after rescaling $L$ by the expression $S^{3} /\langle w\rangle^{2}$, where $S$ represents the standard deviation of the size distribution (see text)

responsible for the distributions here addressed. Therefore, we will not consider it.

Two important aspects must now be addressed to proceed further. First, we notice that in the absence of motion, i.e. in static fields where no collisions can occur, one dune grows only if its neighboring dunes shrink [15], due to sand flux balance and mass conservation. Consequently, if collisions do not occur the inter-dune spacing $L$ between neighboring dunes would scale with the dune size. Our empirical data however, shows a rather different behavior. For each dune field in Fig. 1a-d, the inter-dune spacing $L$ between each dune and its neighbors as a function of the width $w$, distributes parallel to the $w$-axis (Fig. 2b). Thus, contrary to the situation without collisions, here the inter-dune spacing can be taken as its characteristic value, say $\langle L\rangle$, as shown in Fig. 2a and b. This empirical result is a clear sign of a richer internal dynamics in dune fields where collisions play an important role. Indeed, due to collisions, small dunes are continuously emerging from larger ones [13,14], destroying any simple correlation between dune size and inter-dune spacing, and therefore leading to the observed spatial uniformity.

The second aspect is that $\langle L\rangle$ does not follow a simple scaling with the average dune size $\langle w\rangle$ of the field (Fig. 2c)

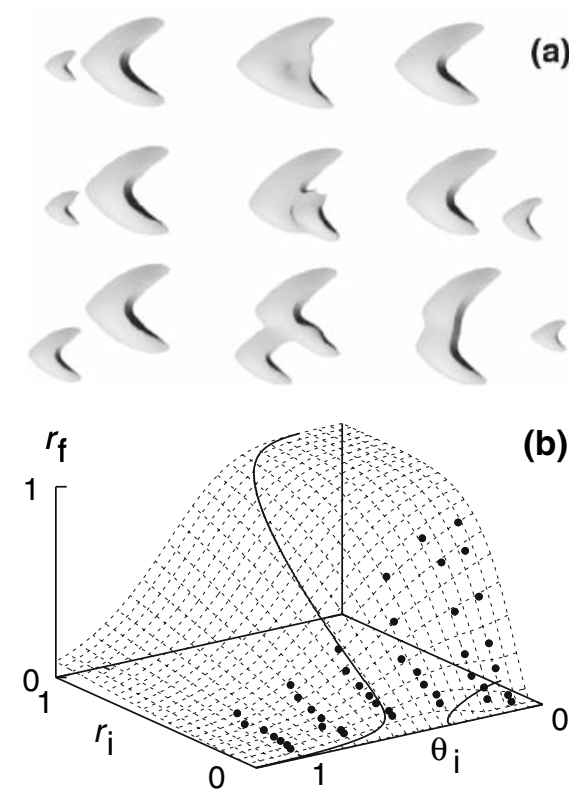

Fig. 3 a Different outcomes of simulated binary collisions, from coalescence (when both dunes merge) on top, to a situation where the volume of the smaller dune increases (decreases) after the collision (middle and bottom sections, respectively). b Collision rule for binary collisions that conserve the number of dunes. Dots represent numerical simulations and dashed lines the corresponding surface fit $r_{\mathrm{f}}\left(r_{\mathrm{i}}, \theta_{\mathrm{i}}\right)$. The curve $r_{\mathrm{f}}\left(r_{\mathrm{i}}, \theta_{\mathrm{i}}\right)=r_{\mathrm{i}}$ (solid line) separates two regimes: one with $r_{\mathrm{f}}>r_{\mathrm{i}}$, where collisions redistribute sand and another with $r_{\mathrm{f}}<r_{\mathrm{i}}$ due to accumulation of sand

but a more complex one (as $S^{3} /\langle w\rangle^{2}$, Fig. 2d) which also involves the standard deviation $S$ of the size distribution. As will be shown later, this scaling naturally emerges from the coupling of binary dune collisions and sand flux balance in the size selection process.

\section{Binary collision dynamics}

With these two empirical findings we proceed by studying the effect of collisions alone in the size distribution of a dune field. Using an established dune model $[1,9,6]$, we simulate ideal binary collisions, under open boundary conditions and constant wind (Fig. 3a), extracting a simple collision rule, i.e. a phenomenological function that relates the relative dune size $r_{\mathrm{f}}$ after the collision between two dunes, and the corresponding initial relative size $r_{\mathrm{i}}$ and offset $\theta_{\mathrm{i}}$ [16]. In accordance to recent underwater experiments [7] and our simulations, for most initial conditions the number of dunes is conserved and the total sand volume change is negligible. Furthermore, as seen in Fig. 3b, in most of the cases the collision increases the relative dune size $\left(r_{\mathrm{f}}>r_{\mathrm{i}}\right)$, redistributing sand from large to small dunes, in sharp contrast to the flux balance, which accumulates sand on large dunes [16]. 


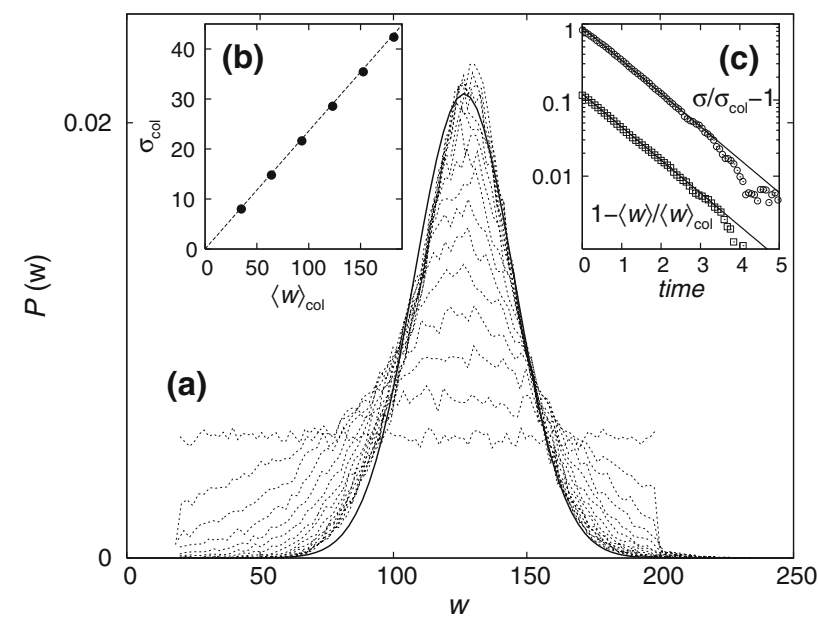

Fig. 4 a Snap-shots of the evolution due to binary collisions of a uniform dune size distribution (dashed lines) towards a Gaussian (solid line). b Linear relation between the two first moments of the observed Gaussian, for different initial volumes of sand available, yielding a constant relative standard deviation $\sigma_{\mathrm{col}} /\langle w\rangle_{\mathrm{col}} . \mathbf{c}$ The first two moments $\langle w\rangle$ (squares) and $\sigma$ (circles), exponentially relax toward their equilibrium values $\langle w\rangle_{\text {col }}$ and $\sigma_{\text {col }}$ (see text). Time units are number of collisions per dune

With this collision rule we study the influence of collisions on the dune size distribution neglecting sand exchange through sand flux between them. A large sampling statistics of about 10,000 dunes is considered, where we assume that each pair of dunes collides with the same fixed probability following the collision rule introduced above (Fig. 3b). This assumption does not consider the spatial distribution of dunes and thus we neglect the spatial correlations between dune sizes and positions. As a result of binary collisions, arbitrary initial size distributions converge to a stationary Gaussian distribution $P_{\text {col }}(w)$, illustrated in Fig. 4a. We find that the relative standard deviation $\sigma_{\mathrm{col}} /\langle w\rangle_{\mathrm{col}}$ of such distribution, where $\langle w\rangle_{\mathrm{col}}$ is the mean size, is constant for different initial conditions (Fig. 4b) and its value is fixed by the parameters of the dune model, which were chosen to reproduce the morphology of Moroccan dunes [6,17], which in turn determine the dune morphology and thus the phenomenological collision rule [16]. The constant value of the relative standard deviation is consequence of the scale invariance of our collision dynamics that depends on the relative dune size instead of the absolute size. On the contrary, with changing wind conditions, the dune scale may influence the collision outcome and also several dunes may emerge from a single collision [14].

Until now, two preliminary conclusions can be stated. First, the measured barchan dunes are approximately uniformly placed over the deserts, with characteristic values of $L$, and the different dune fields follow a common size distribution with a simple scaling with the mean dune size. Second, neither sand flux alone does contribute to the size selection
$[7,11]$ nor collisions alone can be responsible for the skewed measured non-Gaussian distributions (Fig. 1). What results from the interplay between both mechanisms will be now derived from a mean-field approach, leading to a master equation for the size distribution $P(w, t)$ and to a simple scaling between all the three properties, $\langle w\rangle, S$ and $\langle L\rangle$.

\section{Master equation}

When only ideal collisions occur, Fig. 4c shows that both, the mean $\langle w\rangle$ and the standard deviation $\sigma$ of the size distribution exponentially relax toward their respective equilibrium values, $\langle w\rangle_{\mathrm{col}}$ and $\sigma_{\mathrm{col}}$. By using the definition $\langle w\rangle(t)$ $\equiv \int d w w P(w, t)$ and the exponential relaxation $d\langle w\rangle / d t$ $=\left(\langle w\rangle_{\mathrm{col}}-\langle w\rangle\right) / t_{\mathrm{c}}$, the size distribution $P(w, t)$ obeys in first approximation the dynamical equation $d P / d t=\left(P_{\mathrm{col}}-\right.$ $P) / t_{\mathrm{c}}$, where $t_{\mathrm{c}}$ is the characteristic relaxation time (in units of number of collisions per dune) of $P(w)$ towards the equilibrium Gaussian distribution $P_{\text {col }}$ when only collisions are considered.

When both collisions and sand flux balance are considered, the total temporal derivative of $P(w, t)$ has now two separated terms, the partial temporal derivative $\partial P / \partial t$ and the term arising from the volume change rate $\dot{V}=\dot{w} d V / d w$, due to flux balance: $\dot{V} \partial P / \partial V=\dot{w} \partial P / \partial w$. Assuming the existence of a steady state and using the empirical fact that $V \propto w^{3}$ and $\dot{V} \propto Q w$, with $Q$ denoting the saturated sand flux over a flat bed [13], the master-equation yields

$$
\frac{t_{\mathrm{c}}}{t_{\mathrm{s}}} \frac{\partial P(w)}{\partial w}=\frac{w}{\langle w\rangle_{\mathrm{col}}^{2}}\left(P_{\mathrm{col}}(w)-P(w)\right)
$$

where there are three parameters, namely the relative deviation $\sigma_{\mathrm{col}} /\langle w\rangle_{\mathrm{col}}$ of $P_{\mathrm{col}}$, determined by the collision model, the characteristic size $\langle w\rangle_{\mathrm{col}}$ and the ratio $t_{\mathrm{c}} / t_{\mathrm{s}}$. Time $t_{\mathrm{s}}$ is the characteristic time associated to the change rate of the dune size due to the sand flux balance, defined as $t_{\mathrm{s}}=\alpha\langle w\rangle_{\mathrm{col}}^{2} / Q$ with $\alpha$ as a constant. From (1) one concludes that when collisions dominate in the selection of dune sizes, $t_{\mathrm{c}} \ll t_{\mathrm{S}}$ and consequently the distribution converges to the Gaussian $P_{\mathrm{col}}$. When the opposite occurs, with the sand flux balance being the relevant process, $P(w)$ deviates from $P_{\mathrm{col}}(w)$ the more the larger $t_{\mathrm{c}} / t_{\mathrm{s}}$ is.

The solution of (1) is plotted in Fig. 1f, $g$ (solid lines) for each dune field, with $\langle w\rangle_{\mathrm{col}}$ and $t_{\mathrm{c}} / t_{\mathrm{S}}$ as fit parameters. The value of $\sigma_{\text {col }}$ is taken from Fig. $4 \mathrm{~b}$. Apart extreme points, the solution fits well the empirical distributions, with first and second moments reasonable approximated as

$$
\begin{aligned}
& \langle w\rangle \simeq 0.8\langle w\rangle_{\mathrm{col}}\left(\sqrt{t_{\mathrm{c}} / t_{\mathrm{s}}}+1\right) \\
& S /\langle w\rangle \simeq 0.62 \sqrt{t_{\mathrm{c}} / t_{\mathrm{s}}} /\left(\sqrt{t_{\mathrm{c}} / t_{\mathrm{s}}}+1\right)
\end{aligned}
$$


in the range $t_{\mathrm{S}}<5 t_{\mathrm{c}}$. From (2) one sees that the characteristic size $\langle w\rangle_{\text {col }}$ determined by the collisions dynamics is in fact the only characteristic size in the system. Moreover, (2b) shows that the relative standard deviation $S /\langle w\rangle$ is given by the ratio $t_{\mathrm{c}} / t_{\mathrm{s}}$ and thus describes a measure for the competition between sand flux balance $\left(t_{\mathrm{s}}\right)$ and collision $\left(t_{\mathrm{c}}\right)$ processes for the dune size selection. For instance, dune field in Fig. $1 \mathrm{~b}$ has a large value $t_{\mathrm{c}} / t_{\mathrm{s}}=4.3$, indicating that the sand flux balance is the most important size selection process, while the dune field in Fig. $1 \mathrm{c}$ has $t_{\mathrm{c}} / t_{\mathrm{s}}=1.7$ indicating more relevance from collision processes.

Furthermore, the ratio $t_{\mathrm{c}} / t_{\mathrm{S}}$ is not an independent parameter since $t_{\mathrm{c}}$ must be proportional to the collision time $t_{\mathrm{col}}$, defined as the average time for two dunes to collide. This collision time is determined as the quotient between the inter-dune spacing $\langle L\rangle$ and the average relative velocity between two dunes, $\langle v\rangle_{r} \propto \int_{0}^{\infty} d w_{1} \int_{w_{1}}^{\infty} d w_{2} P\left(w_{1}\right) P\left(w_{2}\right)$ $\left(v\left(w_{1}\right)-v\left(w_{2}\right)\right)$. Since the dune velocity follows $v \propto Q / w$ and, within some wide range of sizes, $P(w)$ can be well approximated by a log-normal distribution (see Fig. 1f, g), one

obtains $\langle v\rangle_{r} \propto\langle v\rangle \sigma_{\ell}$ with $\langle v\rangle \propto Q /\langle w\rangle$ the dune average velocity and $\sigma_{\ell}$ the standard deviation of the log-normal distribution (adimensional), yielding $t_{\mathrm{c}} \propto \frac{\langle L\rangle\langle w\rangle}{Q \sigma_{\ell}}$. Substituting $t_{\mathrm{c}}$ and (2a) into (2b) and taking the first-order approximation $\sigma_{\ell} \sim S /\langle w\rangle$, we arrive at

$$
(S /\langle w\rangle)^{3} \simeq a\langle L\rangle /\langle w\rangle \text {. }
$$

As shown in Figs. 1h, i and 2d, where one empirically obtains $\epsilon=(S /\langle w\rangle)^{-1}=2.9 \sqrt[3]{\langle w\rangle /\langle L\rangle}$, the scaling in (3) indeed describes the measurements, with the constant $a=2.9^{-3}$ $=0.041$, independent of the model parameters.

\section{Conclusions}

In summary, due to the dynamical nature of barchan dunes, the size distribution is intrinsically linked to the spatial distribution in such a way that sparse fields have a broader size distribution, while dense ones have narrow distributions. We have shown that the relative dune size distributions of Moroccan dune fields collapse into an unique distribution function, and that dunes are uniformly distributed with a characteristic inter-dune spacing that obeys a simple scaling law. By using a master-equation approach with a simple collision rule, we showed that the simplest processes behind the change of the dune size occurring in dune fields, namely ideal binary collisions and flux balance, are able to properly determine the size distribution function. Which mechanisms are behind the local selection of the specific size scale of a dune field remains an open question, since it involves not only binary collisions and flux balance under a stationary wind, but detailed processes in real changing wind conditions, i.e. calving $[13,14]$, that can locally change the dune size and are not included in the analysis we have presented.

Acknowledgements The authors thank Maria Haase for useful discussions. This research was supported in part by the Max-Planck prize.

Open Access This article is distributed under the terms of the Creative Commons Attribution Noncommercial License which permits any noncommercial use, distribution, and reproduction in any medium, provided the original author(s) and source are credited.

\section{References}

1. Kroy, K., Sauermann, G., Herrmann, H.J.: Minimal model for sand dunes. Phys. Rev. Lett. 88, 054301 (2002)

2. Andreotti, B., Claudin, P., Douady, S.: Selection of dune shapes and velocities. Part 1: dynamics of sand, wind and barchans. Eur. Phys. J. B 28, 321 (2002)

3. Andreotti, B., Claudin, P., Douady, S.: Selection of dune shapes and velocities. Part 2: a two-dimensional modeling. Eur. Phys. J. B 28, 341 (2002)

4. Hersen, P.: On the crescentic shape of barchan dune. Eur. Phys. J. B 37, 507 (2004)

5. Schwämmle, V., Herrmann, H.J.: Solitary wave behaviour of dunes. Nature 426, 619 (2003)

6. Durán, O., Schwämmle, V., Herrmann, H.J.: Breeding and solitary wave behavior of dunes. Phys. Rev. E 72, 021308 (2005)

7. Hersen, P., Douady, S.: Collision of barchan dunes as a mechanism of size regulation. Geophys. Res. Lett. 32, L21403 (2005)

8. Durán, O., Herrmann, H.J.: Vegetation against dune mobility. Phys. Rev. Lett. 97, 188001 (2006)

9. Schwämmle, V., Herrmann, H.J.: A model of barchan dunes including lateral shear stress. Eur. Phys. J. E 16, 57 (2005)

10. Lima, A.R., Sauermann, G., Herrmann, H.J., Kroy, K.: A model for dune fields. Phys A 310, 487 (2002)

11. Hersen, P., Andersen, K.H., Elbelrhiti, H., Andreotti, B., Claudin, P., Douady, S.: Corridors of barchan dunes: stability and size selection. Phys. Rev. E 69, 011304 (2004)

12. Ewing, R.C., Kocurek, G., Lake, L.W.: Pattern analysis of dunefield parameters. Earth Surf. Proc. Landf. 31, 1176-1191 (2006)

13. Elbelrhiti, H., Claudin, P., Andreotti, B.: Field evidence for surface-wave induced instability of sand dunes. Nature 437, 720 (2005)

14. Elbelrhiti, H., Claudin, P., Andreotti, B.: Barchan dune corridors: field characterization and investigation of control parameters. J. Geophys. Res. 113, F02S15 (2008)

15. Kocurek, G., Townsley, M., Yeh, E., Havholm, K., Sweet, M.L. Dune and dune-field development on Padre Island, Texas, with implications for interdune deposition and water-table-controlled accumulation. J. Sedim. Petrol. 62, 622-635 (1992)

16. Durán, O., Schwämmle, V., Herrmann, H.J.: Barchan dune's size distribution induced by collisions. cond-mat/0701370v1

17. Durán, O., Herrmann, H.J.: Modelling of saturated sand flux. J. Stat. Mech. P07011 (2006) 